\title{
HOMEOMORPHIC MEASURES IN METRIC SPACES
}

\author{
JOHN C. OXTOBY
}

\begin{abstract}
ABSTRACr. For any nonatomic, normalized Borel measure $\mu$ in a complete separable metric space $X$ there exists a homeomorphism $h: \Re \rightarrow X$ such that $\mu=\lambda h^{-1}$ on the domain of $\mu$, where $\Re$ is the set of irrational numbers in $(0,1)$ and $\lambda$ denotes Lebesgue-Borel measure in $\Re$. A Borel measure in $\Re$ is topologically equivalent to $\lambda$ if and only if it is nonatomic, normalized, and positive for relatively open subsets.
\end{abstract}

1. Definitions and results. A topological measure space is a pair $(X, \mu)$, where $X$ is a topological space and $\mu$ is a measure on the class of Borel subsets of $X .(X, \mu)$ is homeomorphic to $(Y, \nu)$ if there exists a homeomorphism of $X$ onto $Y$ that makes $\nu$ correspond to $\mu$, and then $\nu$ is said to be topologically equivalent to $\mu$. If $B$ is a Borel subset of $(X, \mu)$, then $\mu_{B}$ denotes the restriction of $\mu$ to the class of Borel subsets of $B$. A measure $\mu$ is everywhere positive if $\mu(G)>0$ for every nonempty open set $G$, nonatomic if $\mu(\{x\})=0$ for each $x \in X$, and normalized if $\mu(X)=1$.

Let $\Re$ denote the set of irrational numbers in $I=[0,1]$, and let $\lambda$ denote the restriction of Lebesgue measure $m$ to the Borel subsets of $\mathfrak{~}$. It is known [8, Theorem 2, p. 886] that a Borel measure in the $n$-dimensional cube $I^{n}$ is topologically equivalent to $n$-dimensional Lebesgue-Borel measure in $I^{n}$ if and only if it is everywhere positive, nonatomic, normalized, and vanishes on the boundary. A similar theorem will be shown to hold in $\Re$.

Theorem 1. A topological measure space $(X, \mu)$ is homeomorphic to $(\Re, \lambda)$ if and only if $X$ is homeomorphic to $\Re$ and $\mu$ is an everywhere positive, nonatomic, normalized Borel measure in $X$. In particular, any such measure in $\boldsymbol{N}$ is topologically equivalent to $\lambda$.

It is known $[2, \S 6$, Exercise $8 \mathrm{c}$, p. 84$]$ that if $X$ is a compact metric space, and $\mu$ is a nonatomic, normalized Borel measure in $X$, then $(X, \mu)$ is almost homeomorphic to $(I, \lambda)$, in the sense that there exist sets $A C I$ and $B C X$ such that $\lambda(I-A)=0, \mu(X-B)=0$, and $\left(B, \mu_{B}\right)$ is homeomorphic to $\left(A, \lambda_{A}\right)$. We shall show that this conclusion still holds when $X$ is a complete separable metric space, and that the set $A$ can always be taken equal to $\Re$.

Received by the editors July 3, 1969.

AMS Subject Classifications. Primary 2813, 2810, 2870; Secondary 5435, 5460.

Key Words and Phrases. Topologically equivalent Borel measures, homeomorphic measure spaces, measure-preserving mapping, complete separable metric space, space of irrational numbers, Cantor set. 
THEOREM 2. If $X$ is a topologically complete separable metric space, and $\mu$ is a nonatomic, normalized Borel measure in $X$, then there exists a $G_{\delta}$ set $B$ in $X$ such that $\mu(X-B)=0$ and $\left(B, \mu_{B}\right)$ is homeomorphic to $(\Re, \lambda)$.

Any uncountable complete separable metric space $X$ contains a copy of $\Re[6$, Corollary 2, p. 352]. Theorem 2 implies that the most general nonatomic, normalized Borel measure in such a space can be constructed by mapping $\mathscr{N}$ into $X$ by a homeomorphism $h$, and then defining $\mu(E)=\lambda h^{-1}(E)$ for every Borel set $E$. The completion of $\mu$ is equal to $m h^{-1}$.

Theorem 2 can be generalized immediately to nonseparable spaces whose separability character has measure zero; such a space has an open set of measure zero whose complement is separable [7, Theorem III, p. 137]. On the other hand, the indispensability of completeness and metrizability is indicated by the following remarks.

REMARK 1. A separable metric space with a nonatomic, normalized Borel measure need not contain a copy of $\mathscr{N}$.

Let $X$ be a subset of $I$ such that both $X$ and $I-X$ meet every nonempty perfect subset of $I$. Then $X$ has outer Lebesgue measure one and inner measure zero. Any relatively Borel subset $A$ of $X$ is of the form $A=X \cap B$, for some Borel set $B$ in $I$. The formula $\mu(A)=m(B)$ defines unambiguously a nonatomic, normalized Borel measure $\mu$ in the separable metric space $X$, but $X$ contains no copy of $\mathscr{N}$.

REMARK 2. A compact Hausdorff space with a nonatomic, normalized, regular Borel measure need not contain a copy of $\mathfrak{N}$, or even of the set $\left\{0,1, \frac{1}{2}, \frac{1}{3}, \cdots\right\}$.

The Stone space $X$ corresponding to any finite nonatomic measure algebra admits a nonatomic, normalized, regular Borel measure $[\mathbf{5}, \S 24]$. Since $X$ is compact and basically disconnected, every infinite closed subset contains a copy of $\beta N$ [4, Problem 9H.2, p. 137], and so its cardinal is at least $2^{c}$. The product of uncountably many copies of $(I, m)$ is another example in which every compact metrizable subspace has measure zero $[2, \S 8$, Exercise $14 a$, p. 110].

2. Proofs of Theorems 1 and 2. A metrizable space is homeomorphic to $\mathfrak{N}$ if and only if it is topologically complete, separable, 0 -dimensional, and nowhere locally compact [1, Satz IV, p. 95]. Hence any nonempty open subset of $\Re$ is homeomorphic to $\Re$. Likewise, any $G_{\delta}$ set that is both dense and frontier in some topologically complete, separable, 0 -dimensional space $Y$ is homeomorphic to $\mathfrak{T}$ [6, Theorem 3, p. 349]. 
Lemma 1. If $\mu$ is an everywhere positive, nonatomic, finite Borel measure in $\Re$, and if $\left\{\alpha_{i}\right\}$ is a sequence of positive real numbers such that $\sum_{1}^{\infty} \alpha_{i}=\mu(\mathfrak{X})$, then there exists a partition of $\mathscr{N}$ into open sets $U_{i}$ such that $\mu\left(U_{i}\right)=\alpha_{i}$ for all $i \in N$.

Proof. Let $a(i, j)=j \alpha_{i} /(j+1)(i \in N, j \in N)$. Let $\left\{r_{k}\right\}(k \in N)$ be an increasing sequence of rational numbers in $(0,1)$, and let $r_{0}=0$. Denote the interval $\left(r_{k-1}, r_{k}\right) \cap \Re$ by $I(i, j)$, where $(i, j)$ is the $k$ th term in the ordering of $N \times N$ defined by $(i, j)<\left(i^{\prime}, j^{\prime}\right)$ if and only if $i+j<i^{\prime}+j^{\prime}$, or $i+j=i^{\prime}+j^{\prime}$ and $j<j^{\prime}$. We wish to determine the sequence $\left\{r_{k}\right\}$ in such a way that

$$
a(i, j)<\sum_{n=1}^{j} \mu(I(i, n))<a(i, j+1)
$$

for all $i$ and $j$. Using the fact that $\mu([0, x] \cap \Re)$ is a strictly increasing continuous map of $[0,1]$ onto $[0, \mu(\mathfrak{N})]$, it is easy to see that such a sequence $\left\{r_{k}\right\}$ can be defined inductively, and that $r_{k}$ will necessarily tend to 1 . Then the sets $U_{i}=\bigcup_{j=1}^{\infty} I(i, j)$ constitute a partition of $\mathfrak{T}$ with the required properties.

Lemma 2. If $\mu$ and $\nu$ are two everywhere positive, nonatomic, Borel measures in $\Re$, if $\rho$ is a metric compatible with the topology of $\mathfrak{N}$, and if $U$ and $V$ are open sets such that $\mu(U)=\nu(V)>0$, then for each $\epsilon>0$ there exist partitions $\left\{U_{i}\right\}$ of $U$ and $\left\{V_{i}\right\}$ of $V$ into nonempty open sets of diameter less than $\epsilon$ such that $\mu\left(U_{i}\right)=\nu\left(V_{i}\right)$ for all $i \in N$.

Proof. Let $\left\{H_{i}\right\}(i \in N)$ be a partition of $V$ into nonempty open sets of diameter less than $\epsilon$. Since $U$ is a copy of $\Re$, by Lemma 1 there exists a partition $\left\{G_{i}\right\}$ of $U$ into open sets such that $\mu\left(G_{i}\right)$ $=\nu\left(H_{i}\right)$ for all $i$. Let $\left\{G_{i j}\right\}(j \in N)$ be a partition of $G_{i}$ into nonempty open sets of diameter less than $\epsilon$. Since $H_{i}$ is a copy of $\Re$ there exists a partition $\left\{H_{i j}\right\}$ of $H_{i}$ into open sets such that $\mu\left(G_{i j}\right)=\nu\left(H_{i j}\right)$ for all $j$. The families $\left\{G_{i j}\right\}$ and $\left\{H_{i j}\right\}$ constitute partitions of $U$ and $V$ having the required properties.

ProOF OF THEOREM 1. It suffices to prove the second assertion. Let $\rho$ be a metric with respect to which $\Re$ is complete. By repeated application of Lemma 2 we obtain partitions $U_{n}=\left\{U\left(i_{1}, \cdots, i_{n}\right)\right\}$ and $V_{n}=\left\{V\left(i_{1}, \cdots, i_{n}\right)\right\}$ of $\mathscr{N}$ into nonempty open sets of $\rho$-diameter less than $1 / n$ such that $U\left(i_{1}, \cdots, i_{n}\right) \supset U\left(i_{1}, \cdots, i_{n+1}\right), V\left(i_{1}, \cdots, i_{n}\right)$ $\supset V\left(i_{1}, \cdots, i_{n+1}\right)$, and $\mu\left(U\left(i_{1}, \cdots, i_{n}\right)\right)=\lambda\left(V\left(i_{1}, \cdots, i_{n}\right)\right)$ for all $n \in N$ and all sets of indices $i_{k} \in N$. For each $x \in \Re$ there is a unique sequence $\left\{i_{n}\right\}$ such that $x \in \bigcap_{n=1}^{\infty} U\left(i_{1}, \cdots, i_{n}\right)$. Define $T(x)$ $=\bigcap_{n=1}^{\infty} V\left(i_{1}, \cdots, i_{n}\right)$. Then $T$ is a 1-1 map of $\Re$ onto itself, and 
$T\left(U\left(i_{1}, \cdots, i_{n}\right)\right)=V\left(i_{1}, \cdots, i_{n}\right)$. Since the union of each of the families $\left\{U_{n}\right\}$ and $\left\{V_{n}\right\}$ is a base with the property that any open set is the union of some disjoint subclass, it follows that $T$ is a homeomorphism and that $\mu(E)=\lambda(T(E))$ for every Borel set $E$ in $\Re$.

Proof of Theorem 2. Let $\left\{x_{i}\right\}$ be a countable dense sequence in $X$. Let $\left\{r_{j}\right\}$ be a sequence of positive real numbers tending to zero such that $\mu\left(\left\{x: \rho\left(x, x_{i}\right)=r_{j}\right\}\right)=0$ for all $i$ and $j$. (Such a sequence exists because for each $i, \mu\left(\left\{x: \rho\left(x, x_{i}\right)=r\right\}\right)>0$ for at most countably many values of $r$.) Let $S_{i j}=\left\{x: \rho\left(x, x_{i}\right)=r_{j}\right\}$ and $U_{i j}=\left\{x: \rho\left(x, x_{i}\right)<r_{j}\right\}$. Then $\left\{U_{i j}\right\}$ is a base for the topology of $X$. Let $S$ be the union of all the sets $S_{i j}$, and let $G$ be the union of all the sets $U_{i j}$ such that $\mu\left(U_{i j}\right)=0$. Then $\mu(G \cup S)=0$, the $G_{\delta}$ set $Y=X-(G \cup S)$ is a topologically complete, separable, 0 -dimensional subspace, and $\mu_{Y}$ is everywhere positive. Let $D$ be a countable dense subset of $Y$, and let $A=G \cup S \cup D$. Then $\mu(A)=0$, and the $G_{\delta}$ set $B=Y-D=X-A$ is both dense and frontier in $Y$. Hence $B$ is homeomorphic to $\Re$, and $\mu_{B}$ is an everywhere positive, nonatomic, normalized Borel measure in $B$. By Theorem $1,\left(B, \mu_{B}\right)$ is homeomorphic to $(\Re, \lambda)$.

3. Uniqueness of $(\mathscr{N}, \lambda)$. Let $F$ denote the class of topological measure spaces $(X, \mu)$, where $X$ is metrizable, separable, and topologically complete (i.e. a Polish space), and $\mu$ is a nonatomic, normalized Borel measure in $X$. The following theorem shows that $(\Re, \lambda)$ is the only member of this class that is topologically contained in each member of the class.

THEOREM 3. If $(X, \mu)$ is a member of $\mathcal{F}$ that is homeomorphic to a subspace of each member of $\mathcal{F}$, then $(X, \mu)$ is homeomorphic to $(\Re, \lambda)$.

Proof. By hypothesis, $(X, \mu)$ is homeomorphic to some subspace $\left(Y, \lambda_{Y}\right)$ of $(\Re, \lambda)$. Since $\lambda(Y)=1, Y$ must be a dense subset of $\Re$. It follows that $Y$ is nowhere locally compact, as well as topologically complete, separable, and 0-dimensional. Consequently $Y$, and therefore $X$, is homeomorphic to $\pi$. Since $\lambda_{Y}$ is everywhere positive, $\mu$ must also be. Therefore $(X, \mu)$ is homeomorphic to $(\Re, \lambda)$, by Theorem 1.

4. Approximation of a Borel set by a Cantor subset. As an application of Theorem 2 we give a new proof of the following theorem, recently proved by Gelbaum [3]. As the referee has pointed out, this theorem is implicitly contained in a result of von Neumann [9, Hilfsatz, p. 577].

Theorem 4. Let $X$ be a complete separable metric space, and let $\mu$ be 
a nonatomic Borel measure in $X$. Any Borel set $A$ in $X$ with $0<\mu(A)$ $<\infty$ is the union of a Cantor set and a set of arbitrarily small measure.

Proof. The formula $\nu(E)=\mu(E \cap A) / \mu(A)$ defines a nonatomic, normalized Borel measure $\nu$ in $X$. By Theorem 2, there exists a set $B$ in $X$ such that $\nu(X-B)=0$ and $\left(B, \nu_{B}\right)$ is homeomorphic to $(\mathfrak{N}, \lambda)$, say by $h$. Then $h(A \cap B)$ is a Borel subset of $\mathfrak{r}$ with $\lambda(h(A \cap B))=1$. Let $C$ be a compact perfect subset of $h(A \cap B)$ with $\lambda(C)>1-\epsilon$. Then $h^{-1}(C) \subset A, \mu\left(A-h^{-1}(C)\right)<\epsilon \mu(A)$, and $h^{-1}(C)$ is compact, perfect, and 0 -dimensional, therefore homeomorphic to the Cantor set.

NOTE ADDED IN PROOF. A result closely related to Theorem 2 is contained in the recent paper by W. Böge, K. Krickeberg, and F. Papangelou, UUber die dem Lebesgueschen Mass isomorphen topologischen Massräume, Manuscripta Math. 1 (1969), 59-77, Corallar, p. 72 .

\section{REFERENCES}

1. P. Alexandroff and P. Urysohn, Über nulldimensionale Punktmengen, Math. Ann. 98 (1928), 89-106.

2. N. Bourbaki, Intégration. Livre VI. Chapitre 5: Intégration des mesures, Hermann, Paris, 1956. MR 18, 881.

3. B. R. Gelbaum, Cantor sets in metric measure spaces, Proc. Amer. Math. Soc. 24 (1970), 341-343.

4. L. Gillman and M. Jerison, Rings of continuous functions, The University Series of Higher Math., Van Nostrand, Princeton, N. J., 1960. MR 22 \#6994.

5. P. R. Halmos, Lectures on Boolean algebras, Van Nostrand Math. Studies, no. 1, Van Nostrand, Princeton, N. J., 1963. MR 29 \#4713. 873.

6. C. Kuratowski, Topologie. Vol. I, 4 ème éd., PWN, Warsaw, 1958. MR 19,

7. E. Marczewski and R. Sikorski, Measures in non-separable metric spaces, Colloq. Math. 1 (1948), 133-139. MR 10, 23.

8. J. C. Oxtoby and S. M. Ulam, Measure-preserving homeomorphisms and metrical transitivity, Ann. of Math. (2) 42 (1941), 874-920. MR 3, 211.

9. J. von Neumann, Einige Sätze ïber Messbare Abbildungen, Ann. of Math. (2) 33 (1932), 574-586.

Bryn Mawr Collegr 